\title{
The nature of B supergiants: clues from a steep drop in rotation rates at $22000 \mathrm{~K}$
} The possibility of bi-stability braking

\author{
Jorick S. Vink ${ }^{1}$, I. Brott ${ }^{2}$, G. Gräfener ${ }^{1}$, N. Langer ${ }^{3}$, A. de Koter ${ }^{2,4}$, and D. J. Lennon ${ }^{5}$ \\ 1 Armagh Observatory, College Hill, Armagh BT61 9DG, Northern Ireland \\ e-mail: jsv@arm.ac.uk \\ 2 Astronomical Institute, Utrecht University, Princetonplein 5, 3584 CC, Utrecht, The Netherlands \\ 3 Argelander-Institut für Astronomie der Universität Bonn, Auf dem Hügel 71, 53121 Bonn, Germany \\ 4 Astronomical Institute Anton Pannekoek, University of Amsterdam, Kruislaan 403, 1098 SJ, Amsterdam, The Netherlands \\ 5 ESA, Space Telescope Science Institute, 3700 San Martin Drive, Baltimore, MD 21218, USA
}

Received 5 February 2010 / Accepted 4 March 2010

\section{ABSTRACT}

\begin{abstract}
The location of B supergiants in the Hertzsprung-Russell diagram (HRD) represents a long-standing problem in massive star evolution. Here we propose their nature may be revealed utilising their rotational properties, and we highlight a steep drop in massive star rotation rates at an effective temperature of $22000 \mathrm{~K}$. We discuss two potential explanations for it. On the one hand, the feature might be due to the end of the main sequence, which could potentially constrain the core overshooting parameter. On the other hand, the feature might be the result of enhanced mass loss at the predicted location of the bi-stability jump. We term this effect "bi-stability braking" and discuss its potential consequences for the evolution of massive stars.
\end{abstract}

Key words. stars: rotation - stars: evolution - stars: early-type - stars: massive - stars: mass-loss -

Hertzsprung Russell and C-M diagrams

\section{Introduction}

The large number of B supergiants as well as their location in the Hertzsprung-Russell diagram represents a long-standing problem in massive star evolution (e.g. Fitzpatrick \& Garmany 1990). Even the most basic question of whether B supergiants are core hydrogen $(\mathrm{H})$ burning main sequence (MS) or helium burning objects has yet to be answered. Here we propose their nature may be revealed utilising their rotational properties.

On the MS, O-type stars are the most rapid rotators known (with $v \sin i$ up to $400 \mathrm{~km} \mathrm{~s}^{-1}$ ), but B supergiants rotate much more slowly (with $v \sin i \lesssim 50 \mathrm{~km} \mathrm{~s}^{-1}$ ), which has been attributed to the expansion of the star after leaving the MS. Hunter et al. (2008) noted a steep drop in rotation rates at low gravities $(\log g<3.2)$ and suggested the slowly rotating B supergiants to be post-MS. The steep drop was also used to constrain the core overshooting parameter $\alpha_{\mathrm{ov}}$ in massive star models (Brott et al. 2010). The slowly rotating B supergiants are also cooler (with $T_{\text {eff }}$ below $\sim 22000 \mathrm{~K}$ ) and $v \sin i$ is observed to drop steeply below this $T_{\text {eff }}$. Here we introduce an alternative explanation for the slow rotation of B supergiants: wind-induced braking due to bi-stability, or bi-stability braking (BSB).

Mass loss plays a crucial role in the evolution of massive stars. Whilst a large amount of attention has been directed towards the role of stellar winds in terms of the loss of mass, as winds "peel off" the star's outer layers (Conti 1976), much less effort has been dedicated to understanding the associated loss of angular momentum (but see Langer 1998; Meynet \& Maeder 2003). Yet the angular momentum aspect of these winds may be equally relevant for understanding massive stars as the loss of mass itself, possibly in a mass range as low as $\sim 10-15 M_{\odot}$.

We first recapture the physics of bi-stable winds and BSB (Sect. 2.1), before presenting the current knowledge of rotational velocities of massive stars. We note a steep drop at $\sim 22000 \mathrm{~K}$ (Sect. 3) and propose two possible explanations for it. In the first one, the drop is due to the separation of MS objects from a second population of slow rotators (Sect. 4.1), whilst in the second one the slow rotation is the result of BSB (Sect. 4.2).

\section{The physics of the mass loss bi-stability jump}

The BS-Jump (Pauldrach \& Puls 1990) is a theoretically predicted discontinuity where wind properties change from a modest $\dot{M}$, fast wind, to a higher $\dot{M}$, slow wind, when the effective temperature drops below $\sim 22000 \mathrm{~K}$. Vink et al. (1999) predicted an increase in the mass-loss rate by a factor of $\sim 5-7$ (see the blue dotted line in Fig. 1) and a drop in the terminal wind velocity by a factor of $\sim 2$. Here the reason for the jump is an increased flux-weighted effective number of iron lines due to the recombination of Fe IV to Fe III (and not necessarily related to the optical depth of the Lyman continuum). In fact, the temperature of the BS-Jump was found to be weakly density dependent, with the BS-Jump starting as high as $\sim 26 \mathrm{kK}$ for the higher mass models and dropping to $\sim 22.5 \mathrm{kK}$ for the lower mass models at $\sim 20 M_{\odot}$ (Vink et al. 2000).

Whilst the predicted drop in terminal wind velocity across the BS range has been confirmed (Crowther et al. 2006), the issue of a jump in mass loss is controversial. The jump may have 


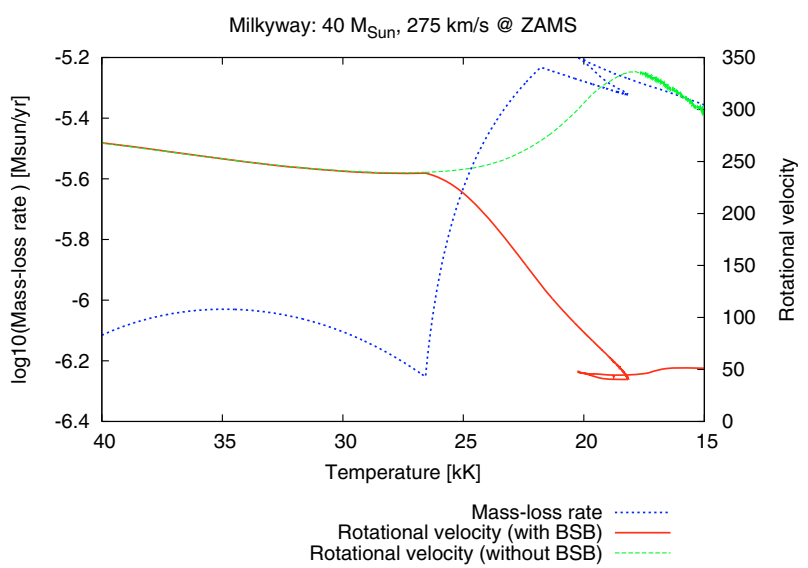

Fig. 1. Mass-loss rate (blue dotted) and rotational velocities for a $40 M_{\odot}$ star with an initial rotational velocity of $275 \mathrm{~km} \mathrm{~s}^{-1}$, including the predicted BS-Jump (red solid) and without it (dashed green). A core overshooting parameter $\alpha_{\mathrm{ov}}$ of 0.335 was employed in these models.

been confirmed in radio data that suggest a local $\dot{M}$ maximum at the predicted temperature (Benaglia et al. 2007), but the predicted rates on the cool side of the jump are up to an order of magnitude larger than those found from state-of-the-art NLTE models (Vink et al. 2000; Trundle \& Lennon 2005; Crowther et al. 2006; Markova \& Puls 2008; Searle et al. 2008). To gain more insight into this mass-loss discrepancy one way forward is to search for other physical effects in the bi-stability region, which might assist us to unravel whether B supergiant mass-loss rates are as high as predicted, or as low as the spectral modelling suggests. Here we outline one such approach, involving stellar rotation rates in the region of the BS-Jump. Vink (2008) pointed out that the temperature of the bi-stability jump coincides with the position where the rotational velocities drop to smaller values (below $100 \mathrm{~km} \mathrm{~s}^{-1}$ ), and suggested this might be due to BSB.

\subsection{Bi-stability braking (BSB)}

Employing stellar evolution models which include mass loss and rotation, we test whether the predicted increase in $\dot{M}$ at the bistability jump might lead to slower rotation. Figure 1 shows the run of the mass-loss rate and predicted rotational velocity as a function of temperature for a $40 M_{\odot}$ star with an initial rotational velocity of $275 \mathrm{~km} \mathrm{~s}^{-1}$. It shows a drastic drop in surface rotation rates for massive stars around $22000 \mathrm{~K}$, which is due to BSB in our models. When we do not increase the mass loss due to the BS-Jump, the stars remain rotating rapidly - despite the stellar expansion, as angular momentum is transferred from the core to the envelope. Bi-stability braking can only be efficient if the star spends a significant amount of time, i.e. part of its MS evolution, on the cool side of the BS-Jump. In our present standard models BSB only occurs above a critical mass of $\sim 30 M_{\odot}$ for the Galaxy, $\sim 35 M_{\odot}$ for the Large Magellanic Cloud (LMC), and $~ 50 M_{\odot}$ for the Small Magellanic Cloud (SMC). In these models we employed a core overshooting parameter $\alpha_{\mathrm{ov}}$ of 0.335 of a pressure scale-height. A higher value of $\alpha_{\text {ov }}$ lowers the critical mass. For instance, in a test calculation with $\alpha_{\mathrm{ov}}=0.5$ at LMC metallicity, BSB occurs already for a $20 M_{\odot}$ star.

\section{The steep drop in rotational velocity at $22000 \mathrm{~K}$}

Howarth et al. (1997) catalogued $v \sin i$ values for 373 OB stars, with roughly half of them being supergiants (luminosity class I;

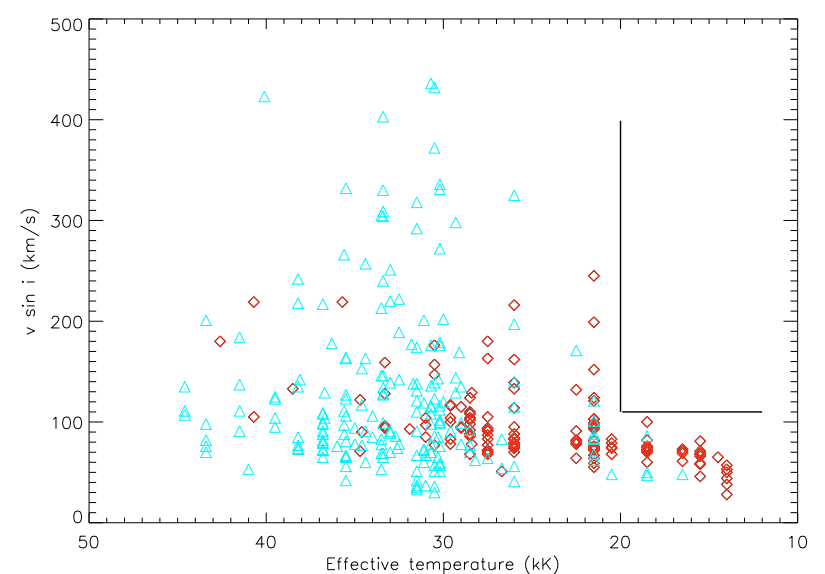

Fig. 2. Projected rotational velocity $v \sin i$ of the Howarth et al. (1997) dataset of Galactic OB supergiants (red diamonds) and non-supergiants (blue triangles) as a function of $T_{\text {eff }}$ (converted from spectral types using Martins et al. 2005; and Crowther et al. 2006). We note that $v \sin i$ drops from values as high as $\sim 400 / 250 \mathrm{~km} \mathrm{~s}^{-1}$ to values below $100 \mathrm{~km} \mathrm{~s}^{-1}$ at $\sim 22 \mathrm{kK}$.

red diamonds). We plot the $v \sin i$ values of this large and uniformly determined data-set in Fig. 2. The figure shows a drop in $v \sin i$ for stars hotter than $22 \mathrm{kK}$ with values as high as $\sim 400 \mathrm{~km} \mathrm{~s}^{-1}$ for all objects and as high as $\sim 250 \mathrm{~km} \mathrm{~s}^{-1}$ for the supergiants only to values that all fall below $100 \mathrm{~km} \mathrm{~s}^{-1}$ for the cooler objects. In other words, we identify a general absence of rapidly rotating B supergiants ${ }^{1}$. As the stars in this data-set have not been analysed in detail, we resort to the results from the FLAMES Survey of massive stars (Evans et al. 2008), which involves data from the Galaxy, the LMC, and the SMC.

Figure 3 shows $v \sin i$ versus effective temperature for the FLAMES data, where we again note a steep drop in $v \sin i$ from values as high as $\sim 400 \mathrm{~km} \mathrm{~s}^{-1}$ to values below $100 \mathrm{~km} \mathrm{~s}^{-1}$. The data selection is a non-trivial undertaking, as we wish to optimise the sample size to sample homogeneity - in the presence of selection effects. The reason we employ a cut-off mass at $15 M_{\odot}$ is that for the largest subset, i.e. that of the LMC, there is a detection limit that runs from $\sim 20 M_{\odot}$ at the hottest temperatures to $\sim 10 M_{\odot}$ at the cool part of the HRD (see Brott et al. 2010). For this reason, we choose an intermediate value of $15 M_{\odot}$ as a minimum value. If we had chosen a higher mass cut-off, the drop feature would shift to a somewhat higher $T_{\text {eff }}$ (up to $27 \mathrm{kK}$ ). If we had opted for a lower mass cut-off, the feature shifts to $T_{\text {eff }}=20 \mathrm{kK}$. Having noted this, tens of manual trials have shown that the sheer drop feature itself does not depend on a particular choice of mass range, and given the presence of the drop in both Figs. 2 and 3, as well as data presented in Fraser et al. (2010), we argue the drop feature is ubiquitous in the entire mass range $\sim 10-60 M_{\odot}$.

The dark grey lines overplotted in Fig. 3 show evolutionary tracks for the intermediate metallicity of the LMC with $v_{\text {rot }}=250 \mathrm{~km} \mathrm{~s}^{-1}$ by Brott et al. (2010) for masses of 15, 20, 30,40 and $60 M_{\odot}$. The black tick-marked dots on the tracks represent evolutionary time-steps of $10^{5}$ years, and are intended to facilitate the comparison with observations.

\footnotetext{
1 Note that the remaining broadness in the B supergiant spectra may (partly) be due to macro-turbulence in addition to, or instead of, rotational broadening (Conti \& Ebbets 1977; Aerts et al. 2009).
} 


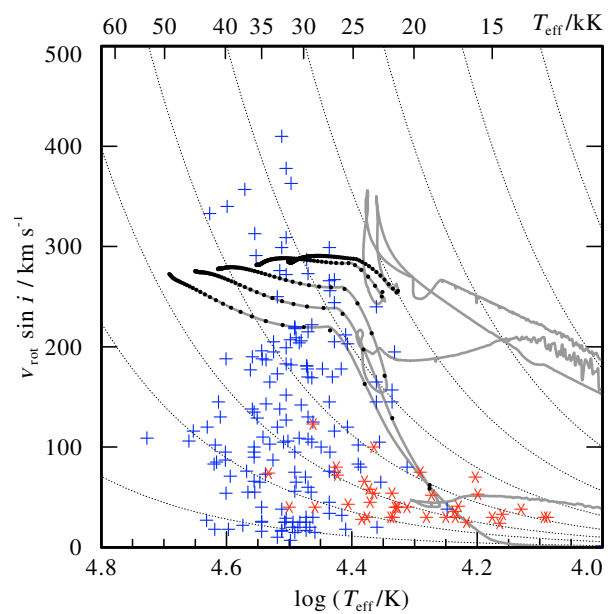

Fig. 3. Rotational velocities vs. $T_{\text {eff }}$ for all FLAMES objects with evolutionary masses above $15 M_{\odot}$. Luminosity classes are shown as blue pluses (luminosity classes II-V) and red stars (luminosity class I). The LMC evolutionary tracks including the predicted BS-Jump are shown in grey with initial $v_{\text {rot }}=250 \mathrm{~km} \mathrm{~s}^{-1}$ for five masses of 15, 20,30, 40 and $60 M_{\odot}$. It can be noted that the critical mass for BSB is $\sim 35 M_{\odot}$ in the LMC. The steepness of these tracks can be compared to the angular momentum conservation case, drawn as grey dotted background lines. The black dots on the tracks represent $10^{5}$ year time-steps.

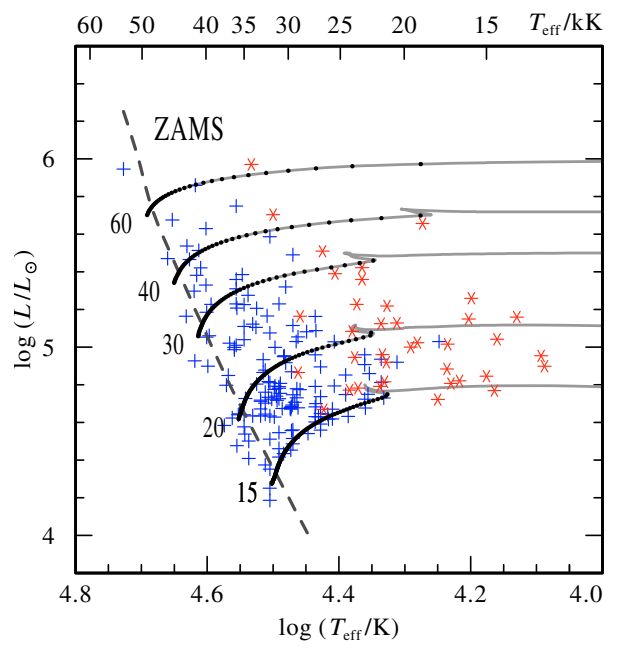

Fig. 4. HRD of the FLAMES survey of massive stars. See the caption of Fig. 3 for an explanation of the symbols.

\section{Two possible interpretations for the drop in $v \sin i$}

In Sect. 3, we highlighted the steep drop in the rotation rates of massive stars, but we have yet to provide an explanation for it. The question is whether the absence of rapidly rotating B supergiants is the result of $\mathrm{BSB}$, or if the cooler slow rotators form an entirely separate population from the hotter MS stars.

\subsection{The case for two populations}

The cool objects (red asterisks) in Fig. 3 and the HRD of Fig. 4 are supergiants of luminosity class I, whilst the fast rotators are dominated by dwarfs (blue pluses). Although it is by no means obvious that supergiants cannot be in a $\mathrm{H}$ burning phase, the division in $\log g$ might imply that we have a population of rapidly rotating MS objects on the one hand, whilst observing a population of slowly rotating evolved supergiants - which have somehow lost their angular momentum - on the other hand. Currently, we

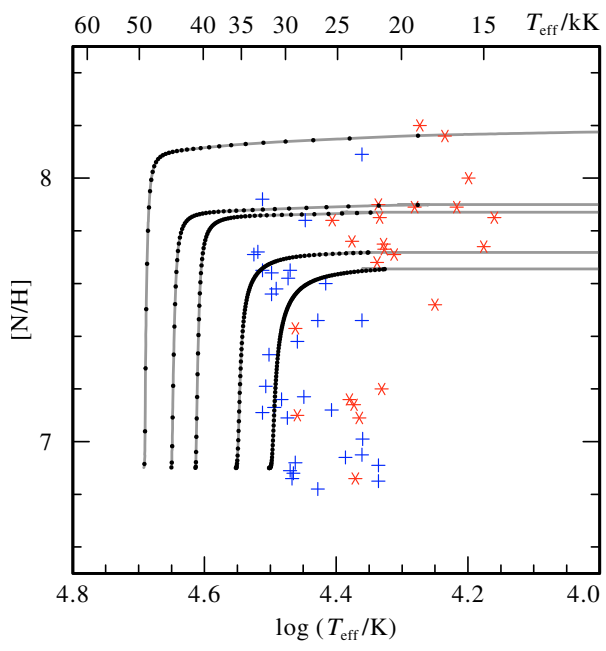

Fig. 5. Nitrogen abundance vs. $T_{\text {eff }}$ for the LMC subset. See the caption of Fig. 3 for an explanation of the symbols.

do not have sufficient information with respect to the evolutionary state of these cool supergiants. In principle, this part of the HRD can be populated with the products of binary evolution, although this would normally not be expected to lead to slowly rotating stars. Alternatively, one could envision the cooler objects to be the product of single star evolution, e.g. post-RSG or blue-loop stars, but the key point is that within the context of the two population interpretation, they are not core $\mathrm{H}$ burning.

A potential distinguishing factor between the "two population scenario" and BSB is that of the chemical abundances. We present LMC N abundances versus effective temperature in Fig. 5, noting that $\mathrm{N}$ abundances could only be derived for a subset of our objects shown in Figs. 3 and 4. As the LMC baseline $[\mathrm{N} / \mathrm{H}]$ equals $\sim 6.9$, the vast majority of slow rotators is found to be strongly $\mathrm{N}$ enhanced. Although rotating models can in principle account for large $\mathrm{N}$ abundances, the fact that such a large number of the cooler objects is found to be $\mathrm{N}$ enriched suggests an evolved nature for these stars.

\subsection{The case for $B S B$}

The second explanation for the steep drop in rotation rates is that both the objects cooler and hotter than $22000 \mathrm{~K}$ reside on the MS, and that it is BSB that explains the slow rotation of the cooler B supergiants. The main argument for BSB is that it is predicted at the temperature where the rotational velocities are found to drop steeply. The evolutionary tracks in Fig. 4 indicate that the MS for the highest mass stars indeed appears rather broad, reaching as far as the BS-Jump temperature at $22 \mathrm{kK}$, and beyond. Therefore, mass loss seems capable of removing a considerable amount of angular momentum during the MS evolution for the highest mass stars.

\section{Discussion}

In principle it is possible that both effects of "two populations" and BSB occur simultaneously at $22000 \mathrm{~K}$, with BSB occurring above a certain critical mass, and the "two population" scenario taking over in the lower mass $\left(10-20 M_{\odot}\right)$ range, but this situation might appear somewhat contrived. The strongest argument for the "two population scenario" are the large N abundances of the B supergiants, whilst the strongest argument for BSB is that 
the drop is observed at the correct location (whilst no such coincidence would be expected for the alternative interpretation).

Using our standard models, BSB can only operate above a certain critical mass and would not be able to explain the steep drop in rotational velocities of stars below the critical mass. The reason BSB does not operate at lower masses in our standard models (of Fig. 3) is that the drop feature has been used to constrain the core overshooting parameter of $\alpha_{\mathrm{ov}}=0.335$. The applicability of BSB could be pushed to lower masses if the MS lifetime were extended. This could be achieved by increasing $\alpha_{\text {ov }}$. When we enlarge $\alpha_{\text {ov }}$ to 0.5 , BSB also occurs at $20 M_{\odot}$ for our Galactic and LMC models. What is clear is that the critical mass is model-dependent. For instance, the solar-metallicity models of Meynet \& Maeder (2003) show BSB in the lower $\left(\sim 15-20 M_{\odot}\right)$ range.

We point out that if BSB were the correct explanation for the drop feature all the way down to $\sim 10 M_{\odot}$, we would require a very large core overshooting parameter, and the consequences would be far-reaching. For instance, it would imply that B (and even A) supergiants are MS objects burning $\mathrm{H}$ in their cores. This would potentially solve the long-standing problem of the presence of such a large number of B supergiants. Moreover, if BSB could work for the entire mass range, it would also have profound implications for the Blue to Red (B/R) supergiant ratio that has been used to constrain massive star models as a function of metallicity for decades. Furthermore, if the absence of rapidly rotating $\mathrm{B}$ supergiants is due to $\mathrm{BSB}$, one might wonder what this would imply for the evolutionary state of the presumably rapidly rotating $\mathrm{B}[\mathrm{e}]$ supergiants. The rapid rotation of these extreme objects could possibly be related to close binary evolution or merging (Pasquali et al. 2000), but this requires future investigation.

If BSB would indeed occur in the lower mass range (down to $\sim 10 M_{\odot}$ ), one should be aware that the derived overshooting parameter of 0.335 becomes a lower limit and that the real value becomes larger. Although this would be consistent with the suggested increase in $\alpha_{\text {ov }}$ with stellar mass (Ribas et al. 2000), such a large value of $\alpha_{\text {ov }}$ might be considered uncomfortable, as the highest mass data-point in Ribas et al. is based on one binary star, V380 Cyg, for which the results have been challenged (Claret 2003).

To summarise, we have presented two potential explanations for the steep drop in rotation rates at $22000 \mathrm{~K}$. Currently, we have insufficient information to decide which one is correct. In any case, our study demonstrates the important role of mass loss for massive star evolution, and especially the importance of specifics in its dependence on the stellar parameters. Furthermore, we have highlighted the significant influence of mass loss on the angular momentum transport in massive stars. Last but not least, BSB may offer a novel method of diagnosing the effects of mass loss via its influence on the angular momentum. Current analyses yield controversial results with respect to the existence of a BS jump. On the one hand, the predicted drop in terminal wind velocity across the BS range has been confirmed (Crowther et al. 2006). On the other hand, for temperatures below the BS-Jump, the mass-loss rates obtained from spectral modelling are generally much lower than predicted (Vink et al. 2000; Crowther et al. 2006).

A simultaneous investigation of the abundances, mass loss, and rotational properties of a large sample of massive stars, e.g. with the FLAMES II Tarantula survey (Evans et al. 2010), would be most helpful to settle these issues.

\section{References}

Aerts, C., Puls, J., Godart, M., \& Dupret, M.-A. 2009, A\&A 508, 409 Benaglia, P., Vink, J. S., Marti, J., et al. 2007, A\&A, 467, 1265

Claret, A. 2003, A\&A, 399, 1115

Conti, P. S. 1976, MSRSL, 9, 193

Conti, P. S., \& Ebbets, D. 1977, ApJ, 213, 438

Crowther, P. A., Lennon, D. J., \& Walborn, N. R. 2006, A\&A, 446, 279

Evans, C., Hunter, I., Smartt, S., et al. 2008, Msngr, 131, 25

Evans, C. J., Bastian, N., Beletsky, Y., et al. 2010, in Star clusters: basic galactic building blocks throughout time and space, Proc. IAU Symp., 266, 35 [arXiv: 0909.1652]

Fitzpatrick, E. L., \& Garmany, C. D. 1990, ApJ, 363, 119

Fraser, M., Dufton, P. L., Hunter, I., \& Ryans, R. S. I. 2010, MNRAS, submitted, [arXiv: 1001.3337$]$

Howarth, I. D., Siebert, K. W., Hussain, G. A. J., \& Prinja, R. K. 1997, MNRAS, 284,265

Hunter, I., Lennon, D. J., Dufton, P. L., et al. 2008, A\&A, 479, 541

Langer, N. 1998, A\&A, 329, 551

Markova, N., \& Puls, J. 2008, A\&A, 478, 823

Martins, F., Schaerer, D., \& Hillier, D. J. 2005, A\&A, 436, 1049

Meynet, G., \& Maeder, A. 2003, A\&A, 404, 75

Pasquali, A., Nota, A., Langer, N., et al. 2000, AJ, 119, 1352

Pauldrach, A. W. A., \& Puls, J. 1990, A\&A, 237, 409

Ribas, I., Jordi, C., \& Gimenez, A. 2000, MNRAS, 318, 55

Searle, S. C., Prinja, R. K., Massa, D., \& Ryans, R. 2008, A\&A, 481, 777

Trundle, C., \& Lennon, D. J. 2005, A\&A, 434, 677

Vink, J. S. 2008, IAU Symp. 252, 271 [arXiv: 0809.4789]

Vink, J. S., de Koter, A., \& Lamers, H. J. G. L. M. 1999, A\&A, 350, 181

Vink, J. S., de Koter, A., \& Lamers, H. J. G. L. M. 2000, A\&A, 362, 295 